\title{
GM-CSF Expression and Macrophage Polarization in Joints of Undifferentiated Arthritis Patients Evolving to Rheumatoid Arthritis or Psoriatic Arthritis
}

\section{Sara Fuentelsaz-Romero}

Hospital General Universitario Gregorio Maranon

\section{Andrea Cuervo}

Hospital Clinic de Barcelona

\section{Lizbeth Estrada-Capetillo}

Universidad Autonoma de Ciudad Juarez

\section{Raquel Celis}

Hospital Clinic de Barcelona

\section{Raquel García-Campos}

Hospital General Universitario Gregorio Maranon

Julio Ramírez

Hospital Clinic de Barcelona

\section{Sergi Sastre}

Hospital Clinic de Barcelona

\section{Rafael Samaniego}

Hospital General Universitario Gregorio Maranon

\section{Amaya Puig-Kröger}

Hospital General Universitario Gregorio Maranon

Juan D Cañete ( $\boldsymbol{\nabla}$ jcanete@clinic.cat)

Hospital Clinic i Provincial https://orcid.org/0000-0003-2606-0573

\section{Research article}

Keywords: GM-CSF, macrophages, synovial tissue, undifferentiated arthritis, psoriatic arthritis, rheumatoid arthritis

Posted Date: June 15th, 2020

DOI: https://doi.org/10.21203/rs.3.rs-34812/v1

License: (c) (1) This work is licensed under a Creative Commons Attribution 4.0 International License. Read Full License 


\section{Abstract}

Background and Aims: GM-CSF-dependent macrophage polarization hasbeen demonstrated in rheumatoid arthritis (RA). Our aim was to seek diagnostic/prognostic biomarkers for undifferentiated arthritis (UA) by analyzing GM-CSF expression and source, macrophage polarization and density in joints of patients with UA evolving to RA or PsA compared with established RA or PsA, respectively.

Methods:Synovial tissue (ST)from patients with UA evolving to RA (UA>RA, $n=8), P s A(U A>P s A, n=9$ ), persistent UA (UA, n=16), established RA ( $n=12)$ and PsA $(n=10)$, and healthy controls $(n=6)$, were analyzed. Cell source and quantitative expression of GM-CSF and proteins associated with pro-inflammatory (GM-CSFdriven) and anti-inflammatory (M-CSF-driven) macrophage polarization (activin A, TNFa, MMP12 and CD209, respectively) were assessed in $\mathrm{ST} \mathrm{CD}_{163^{+}}$macrophages by multicolor immunofluorescence. GM-CSF and activin $A$ levels were also quantified in paired synovial fluid samples. $C D 163^{+}$macrophage density was determined in all groups by immunofluorescence.

Results:Synovial stromal cells ( $\mathrm{FAP}^{+}$fibroblast, $\mathrm{CD} 90^{+}$endothelial cells) and $\mathrm{CD} 163^{+}$sublining macrophages were the sources of GM-CSF. ST CD $163^{+}$macrophages from all groups expressed pro-inflammatory polarization markers (activin A, TNFa and MMP12). Expression of the M-CSF-dependent anti-inflammatory marker CD209 identified two macrophage subsets (CD163 ${ }^{+}$CD209high and CD163 ${ }^{+}$CD209low/ $)$. CD 209 ${ }^{+}$ macrophages were more abundant in ST from healthy controls and PSA patients, although both macrophage subtypesshowed similar levels of pro-inflammatory markers in all groups. In paired synovial fluid samples, activin A was detected in all patients, with higher levels in UA>RA and RA, while GM-CSF was infrequently detected. ST CD163+ macrophage density was comparable between UA>RA and UA>PsA patients, but significantly higher than in persistent UA and established RA and PsA patients, respectively.

Conclusions: GM-CSF is highly expressed by sublining $\mathrm{CD} 90^{+} \mathrm{FAP}^{+}$synovial fibroblasts, $\mathrm{CD} 90^{+}$activated endothelium and $\mathrm{CD} 163^{+}$macrophages in different types of arthritis. The polarization state of ST macrophages was similar in all UA and established arthritis groups, with a predominance of proinflammatory GM-CSF-associated markers. CD163+ macrophage density was significantly higher in the UA phases of RA and PsA compared with persistent UA. Taken together, our findings support the idea that GMCSF is a strong driver of macrophage polarization and a potential therapeutic target not only in RA but also in PsA and all types of UA.

\section{Introduction}

Undifferentiated arthritis (UA) is frequent in early arthritis clinics, with a prevalence of $30-50 \%$ [1-3]. After one year of follow up, approximately $30 \%$ of patients progress to rheumatoid arthritis (RA) and $7-15 \%$ to peripheral spondyloarthritis (pSpA), mainly to psoriatic arthritis (PsA) [4]. SpA is a concept encompassing a group of musculoskeletal inflammatory diseases (axial spondyloarthritis, peripheral SpA, PsA, reactive arthritis, arthritis associated with inflammatory bowel disease) sharing immunogenetic, clinical and radiographic characteristics. PsA is the most prevalent peripheral SpA and is characterized by skin and musculoskeletal inflammation (arthritis, enthesitis, dactylitis and spondylitis) $[5,6]$. 
RA and peripheral PsA are chronic inflammatory diseases mediated by the immune system and, although joint damage may be early and frequent in both diseases if not treated, their pathogenesis is different with respect to the relative participation of the adaptive and innate immune systems. This is shown by therapies that are useful in treating RA (anti-CD20, rituximab; CTL4-IgG, abatacept; anti- IL6 R, tocilizumab) but not PsA. Inversely, therapies inhibiting the IL-17/IL-23 pathway are successful in treating PsA, but do not function in RA patients [7].

The search for biomarkers enabling early classification of UA patients, earlier initiation of specific therapy and better outcomes has recently increased interest in the study of inflamed synovial tissue (ST) or synovitis, the primary target of inflammatory joint diseases [8]. Synovitis in RA and PsA, the two most-frequent definite diagnoses in UA, share multiple similarities in the composition and structural distribution of their cellular infiltrates [9]. $\mathrm{CD}^{+} 8^{+}$macrophages accumulate in the synovium of RA and PsA joints, where they show destructive and remodeling potential and contribute considerably to joint inflammation and damage [10,11]. In fact, RA and PsA macrophage density correlates with disease activity $[12,13]$. Sublining CD68 ${ }^{+}$ macrophage density has been shown to be similar in PsA and RA synovitis [14] although, in a small study we found that $\mathrm{CD} 68^{+}$macrophage density was associated with erosive disease only in RA, suggesting that the destructive potential of ST CD68 $8^{+}$macrophages may differ between RA and PsA [15].

Studies have analyzed macrophage subsets in chronic arthritis with differing results, probably due to the markers used. Hemoglobin/haptoglobin scavenger receptor CD163-positivity was proposed as a biomarker of anti-inflammatory macrophages because it was overexpressed in peripheral SpA, including PsA synovitis. However, there was overexpression of pro-inflammatory macrophage markers in RA [16]. By using surface markers (CD14, CD163, CD68, CD32, CD64, CD200R, CD80) on ST macrophages from RA and SpA, including PsA patients, this study found that macrophages had a mixed M1-proinflammatory/M2-anti-inflammatory phenotype, with M1 predominance in RA and IL-10-expressing macrophages in SpA [16]. More recently, the molecular characterization of GM-CSF-dependent (pro-inflammatory) and M-CSF-driven (anti-inflammatory) monocyte-derived macrophages [17-19] has allowed the definition of the polarization state of macrophages in RA. Specifically, CD $163^{+}$RA ST macrophages have a high expression of GM-CSF-associated markers (MMP12, EGLN3, INHBA, TNFa and CCR2) but low levels of the M-CSF-associated marker CD209 [17-19].

Given the putative pathological role of GM-CSF and M-CSF in synovitis [20-22], our aims were to seek diagnostic/prognostic biomarkers by analyzing potential differences in GM-CSF expression and its cellular source and CD163 + macrophage polarization and density in joints of patients with UA evolving to RA or PsA compared with persistent UA and established RA or PsA, respectively.

\section{Methods}

\section{Patients}

Patients with UA and synovial biopsies were retrospectively selected when they met the classification criteria for RA or PsA during follow up. Synovial biopsies obtained by rheumatologic arthroscopy [9] from inflamed knee joints of patients with active persistent UA synovitis $(U A, n=16), U A$ evolving to RA (UA>RA, $n=8)$ and 
UA evolving to PsA (UA > PsA, $n=9$ ), together with synovial biopsies from patients fulfilling the American College of Rheumatology (ACR) criteria for RA [23] $(n=12)$ or the CASPAR criteria for PsA [24] $(n=10)$ as positive controls, were included. ST from 6 healthy controls (4 men, 2 women, aged $36 \pm 5$ years) undergoing arthroscopic meniscectomy, were included. No patient had received biological therapy at biopsy. Arthroscopy was performed under diagnostic and/or therapeutic (lavage) indications with a $2.7 \mathrm{~mm}$ arthroscope (Storz, Tullingen, Germany). Eight samples were obtained from the suprapatellar pouch and the medial and lateral gutters in each patient [9]. The study was approved by the Ethics Research Committee of the Hospital Clinic of Barcelona, Spain (HCB/2014/0579) and signed informed consent was obtained from each patient.

\section{Immunofluorescence and multicolor confocal microscopy}

Multicolor confocal microscopy was performed as previously described [17]. Briefly, the following antibodies were used: FITC-labelled anti-CD163 (Ber-Mac3, MBL International Corp., MA, USA), anti-M-CSFR/CD115 (AF329, R\&D Systems), anti-TNFa (ab6671, Abcam), anti-MMP12 (ab66157, Abcam), anti-INHBA (ab97705, Abcam), anti-CD209 (MR1; kindly provided by Angel L. Corbí, Centro de Investigaciones Biológicas, Madrid, Spain), anti-CD90 (E510, BD Pharmingen), anti-FAP (ab65398, Abcam), anti-GM-CSF (sc-13101, Santa Cruz Biotechnology), anti-CD3 (SK7) and anti-CD4 (SK3), from BD Biosciences, isotype-matched control antibodies and fluorochrome-conjugated secondary antibodies (Jackson Immunoresearch). Tissues samples were snapfrozen in OCT, and $4 \mu \mathrm{m}$ cryosections were blocked for 10 min with $1 \%$ human immunoglobulins and incubated with primary $(1-5 \mu \mathrm{g} / \mathrm{ml})$ and appropriate secondary antibodies. Imaging was performed using an inverted confocal microscope (SPE, Leica Microsystems) and an ACS-APO 20x/NA 0.60 glycerol immersion objective. Similar acquisition settings were used for all samples. Considering that fluorescence intensity correlated with molecules on the surface of the cell, the mean number of molecules of every single cell (Mean Fluorescence Intensity, MFI) was calculated using ImageJ/FIJI software (National Institutes of Health, Bethesda, MD, USA). All quantifications were blinded and at least three random fields next to the lining layer were evaluated for each type of tissue, quantifying the expression of activin A, TNF-a, MMP12 and CD209 in all segmented $\mathrm{CD}_{163^{+}}$macrophages. CD115 and/or CD163 macrophage pan-markers were used to segment these cells in tissues and estimate the mean intensity of the proteins of interest. Pan-markers were intentionally acquired in saturated conditions to better depict macrophages areas. Macrophage density was normalized based on the selected tissue area $\left(\mathrm{mm}^{2}\right)$. After background subtraction, data were plotted using GraphPad software (GraphPad Software, La Jolla, CA, USA).

\section{Macrophage culture}

Human peripheral blood mononuclear cells (PBMC) were isolated from buffy coats from normal donors over a Lymphoprep (Nycomed Pharma) gradient. Monocytes were purified from PBMC by magnetic cell sorting using CD14 microbeads (Miltenyi Biotech). Monocytes were cultured at $0.5 \times 10^{6} \mathrm{cells} / \mathrm{ml}$ for 7 days in RPMI 1640 supplemented with $10 \%$ fetal calf serum, at $37^{\circ} \mathrm{C}$ in a humidified atmosphere with $5 \% \mathrm{CO}_{2}$, and containing GM-CSF (1000 U/ml) to generate GM-CSF-polarized macrophages (termed GM-M $\varnothing$ ), or M-CSF (10 ng/ml) for M-CSF-polarized macrophages (termed M-MØ). Cytokines were added every two days.

\section{Flow cytometry}


Phenotypic analysis was carried out by immunofluorescence using standard procedures. Mouse monoclonal antibodies used for cell-surface staining included FITC-labeled anti-CD14 (Miltenyi), anti-CD115 (R\&D Systems) and FITC-labeled anti-CD163 (MBL International Corp., MA). Alexa Fluor-647-labeled isotypematched secondary antibody (Jackson Immunoresearch) was used to stain anti-CD115. Control $\mathrm{Y}_{1} \mathrm{FITC} / \mathrm{Y}_{2 a}$ PE (BD) was included as a negative control.

\section{Quantitative real time RT-PCR and GSEA}

Total RNA was retrotranscribed and cDNA was quantified using the Universal Human Probe Roche library (Roche Diagnostics). Quantitative real-time PCR (qRT-PCR) was performed on a LightCycler ${ }^{\circledR} 480$ (Roche Diagnostics). Assays were made in triplicate and results normalized according to the expression levels of TBP. The results were obtained using the $\triangle \triangle C T$ method for quantitation. The oligonucleotides used to quantify mRNA transcripts were (5'-3'): CD209s cagagtggggtgacatgagtgac; CD209as gtgaagttctgctacgcaggag; INHBAs ctcggagatcatcacgtttg; INHBAas ccttggaaatctcgaagtgc; MMP12s tgtcactaccgtgggaaataag; MMP12as aacactggtctttggtctctcag; TBPs cggctgtttaacttcgcttc; TBPas cacacgccaagaaacagtga; TNFas cagcctcttctccttcctgat; TNFaas gccagagggctgattagaga. For Gene Set Enrichment Analysis (GSEA) (http://software.broadinstitute.org/gsea/index.jsp) [25], the gene signature from RA synovial macrophages versus monocyte-derived macrophages limma analysis of the microarray data in GSE10500 was used [26]. The previously defined GM-M Ø-specific markers data set (from GSE68061) was used for GSEA [27].

\section{Elisa}

GM-CSF (Human GM-CSF ELISA MAX Deluxe, Biolegend) and activin A (Human Activin A DuoSet ELISA, R\&D Systems) were quantified using commercially-available ELISA.

\section{Statistical Analysis}

The Mann Whitney test and Student's $t$ test were used and Spearman's correlation was determined. A value of $p<0.05$ was considered statistically significant $\left({ }^{*}, p<0.05 ;{ }^{* *}, p<0.01 ;{ }^{* * *}, p<0.001\right)$. The analysis was made using GraphPad Prism software.

\section{Results}

\section{Clinical and demographic features}

Clinical, demographic and serologic data of the patients included are detailed in Table 1. UA > RA patients had significantly fewer swollen and tender joints, and a lower DAS28, than established RA patients. UA > PsA patients were significantly younger than those with established PsA. No significant between-group differences were found with respect to systemic inflammation biomarkers. Some patients were taking csDMARDs, generally methotrexate, at arthroscopy. A high percentage of patients did not receive csDMARDs, due to knee involvement or because they had few inflamed joints, which were treated with NSAIDs or local 
infiltration with glucocorticoids in the 3 months before arthroscopy. No patient was treated with biological therapy before arthroscopy. (Table 1). 
Table 1

Clinical and demographic data.

\begin{tabular}{|c|c|c|c|c|c|c|c|c|}
\hline & UA & $U A>R A$ & UA $>$ PsA & $\mathrm{RA}$ & PsA & $\begin{array}{l}\text { UA> } \\
\text { RA vs } \\
\text { UA> } \\
\text { PsA }\end{array}$ & $\begin{array}{l}\text { UA > } \\
\text { RA vs } \\
\text { RA }\end{array}$ & $\begin{array}{l}\text { UA> } \\
\text { PsA } \\
\text { vs } \\
\text { PsA }\end{array}$ \\
\hline & $n=16$ & $n=8$ & $n=9$ & $n=12$ & $n=10$ & $\mathrm{p}$ & $p$ & $\mathrm{p}$ \\
\hline $\begin{array}{l}\text { Age } \\
\text { (years) }\end{array}$ & $\begin{array}{l}57 \\
(40- \\
65)\end{array}$ & $56(42-71)$ & $44(35-49)$ & $58(52-71)$ & $53(48-62)$ & 0.102 & 0.643 & 0.024 \\
\hline $\begin{array}{l}\text { Sex } \\
\text { (male) } \\
\text { n(\%) }\end{array}$ & $5(31)$ & $3(38)$ & $5(56)$ & $3(25)$ & $8(80)$ & 0.457 & 0.550 & 0.252 \\
\hline $\begin{array}{l}\text { Disease } \\
\text { duration } \\
\text { until } \\
\text { synovial } \\
\text { biopsy } \\
\text { (months) }\end{array}$ & $\begin{array}{l}30 \\
(21- \\
37)\end{array}$ & $13(6-51)$ & $6(3-30)$ & $\begin{array}{l}104(73- \\
202)\end{array}$ & $\begin{array}{l}129(45- \\
265)\end{array}$ & 0.289 & 0.002 & 0.003 \\
\hline $\begin{array}{l}\text { Follow-up } \\
\text { until } \\
\text { definite } \\
\text { diagnosis } \\
\text { (months) }\end{array}$ & $\begin{array}{l}32 \\
(11- \\
59)^{\star}\end{array}$ & $1(0.5-7)$ & $1(0.5-2)$ & NA & NA & 0.847 & NA & NA \\
\hline $\begin{array}{l}\text { Disease } \\
\text { duration } \\
\text { (months) }\end{array}$ & $\begin{array}{l}60 \\
(36- \\
97)\end{array}$ & $34(7-57)$ & $6(4-36)$ & $\begin{array}{l}28(10- \\
82)\end{array}$ & $\begin{array}{l}25(12- \\
56)\end{array}$ & 0.177 & 0.671 & 0.060 \\
\hline SJC & $\begin{array}{l}1(1- \\
2)\end{array}$ & $2(1-9)$ & $1(1-2)$ & $11(4-19)$ & $1(1-1)$ & 0.141 & 0.033 & 0.850 \\
\hline TJC & $\begin{array}{l}1(1- \\
2)\end{array}$ & $3(2-5)$ & $1(1-2)$ & $19(4-25)$ & $1(1-1)$ & 0.009 & 0.015 & 0.633 \\
\hline $\begin{array}{l}\text { CRP } \\
\text { basal } \\
\text { (mg/dl) }\end{array}$ & $\begin{array}{l}1.34 \\
(0.57- \\
2.53)\end{array}$ & $\begin{array}{l}2.46(0.51- \\
3.12)\end{array}$ & $\begin{array}{l}1.70(0.39- \\
8.23)\end{array}$ & $\begin{array}{l}4.45(1.20- \\
10.10)\end{array}$ & $\begin{array}{l}3.55(0.64- \\
7.87)\end{array}$ & 0.630 & 0.117 & 0.744 \\
\hline $\begin{array}{l}\text { ESR basal } \\
(\mathrm{mm} / \mathrm{h})\end{array}$ & $\begin{array}{l}17(7- \\
32)\end{array}$ & $34(20-67)$ & $30(12-40)$ & $49(22-69)$ & $8(5-41)$ & 0.499 & 0.589 & 0.204 \\
\hline $\begin{array}{l}\text { DAS28 } \\
\text { basal } \\
\text { (VSG) }\end{array}$ & $\begin{array}{l}3.34 \\
(2.68- \\
3.82)\end{array}$ & $\begin{array}{l}4.40(3.43- \\
5.39)\end{array}$ & $\begin{array}{l}3.73(2.85- \\
4.17)\end{array}$ & $\begin{array}{l}5.84(4.88- \\
7.59)\end{array}$ & $\begin{array}{l}3.13(2.27- \\
4.24)\end{array}$ & 0.102 & 0.039 & 0.391 \\
\hline
\end{tabular}

Data are expressed as median (Interquartil range). Mann Whitney test, $T$ student

UA: persistent undifferentiated arthritis; UA > RA: undifferentiated arthritis evolving to RA; UA > PsA: undifferentiated arthritis evolving to PSA; RA: rheumatoid arthritis; PsA: psoriatic arthritis; SJC swollen joint count, TJC: tender joint count, CRP: C-reactive protein; ESR: erythrocyte sedimentation rate; DAS: Disease Activity Score;RF: rheumatoid factor, ACPA: anti-citrullinated protein antibody; csDMARD: conventional synthetic Drugs Modifying NA: not applicable; $p$ : $p$ value; *Follow-up in persistent UA group, without diagnosis. 


\begin{tabular}{|c|c|c|c|c|c|c|c|c|}
\hline & UA & $\mathrm{UA}>\mathrm{RA}$ & $\mathrm{UA}>\mathrm{PsA}$ & RA & PsA & $\begin{array}{l}\text { UA > } \\
\text { RA vs } \\
\text { UA > } \\
\text { PsA }\end{array}$ & $\begin{array}{l}\text { UA> } \\
\text { RA vs } \\
\text { RA }\end{array}$ & $\begin{array}{l}\text { UA > } \\
\text { PsA } \\
\text { vs } \\
\text { PsA }\end{array}$ \\
\hline $\begin{array}{l}\mathrm{RF} \text { and/or } \\
\text { ACPA } \\
\mathrm{n}(\%)\end{array}$ & 0 & $4(50)$ & 0 & $9(75)$ & 0 & 0.006 & 0.710 & - \\
\hline $\begin{array}{l}\text { csDMARD } \\
\mathrm{n}(\%)\end{array}$ & $7(44)$ & $2(25)$ & $6(67)$ & $6(50)$ & $2(20)$ & 0.205 & 0.667 & 0.040 \\
\hline \multicolumn{9}{|c|}{ Data are expressed as median (Interquartil range). Mann Whitney test, $T$ student } \\
\hline \multicolumn{9}{|c|}{$\begin{array}{l}\text { UA: persistent undifferentiated arthritis; UA > RA: undifferentiated arthritis evolving to RA; UA > PsA: } \\
\text { undifferentiated arthritis evolving to PSA; RA: rheumatoid arthritis; PsA: psoriatic arthritis; SJC swollen } \\
\text { joint count, TJC: tender joint count, CRP: C-reactive protein; ESR: erythrocyte sedimentation rate; DAS: } \\
\text { Disease Activity Score;RF: rheumatoid factor, ACPA: anti-citrullinated protein antibody; csDMARD: } \\
\text { conventional synthetic Drugs Modifying NA: not applicable; p: pvalue; *Follow-up in persistent UA group, } \\
\text { without diagnosis. }\end{array}$} \\
\hline
\end{tabular}

\section{Synovial tissue stromal cells and CD163 + macrophages are the main source of GM-CSF in undifferentiated and established arthritis.}

To assess GM-CSF levels in the undifferentiated phases of human arthritis, we first determined GM-CSF expression in the ST of UA, UA > RA, UA > PsA, established RA and PsA patients, and healthy controls (Table 1). Except in healthy ST, GM-CSF was readily detectable in all groups (Fig. 1A). Immunofluorescence analysis revealed $\mathrm{GM}-\mathrm{CSF}$ in $\mathrm{CD} 90^{+}$activated endothelium, $\mathrm{CD} 90^{+} \mathrm{FAP}^{+}$fibroblasts from the sublining layer around the endothelium, and in sublining $\mathrm{CD}_{163^{+}}$macrophages (Fig. 1A). However, GM-CSF was not found in $\mathrm{CD}^{+}$or $\mathrm{CD} 4+\mathrm{T}$ cells (data not shown).

These results indicate that GM-CSF is detected similarly in the earlier and established stages of RA and PsA, and in persistent $\mathrm{UA}$, and that GM-CSF-producing cells are pathogenic $\mathrm{CD} 90^{+} \mathrm{FAP}^{+}$synovial fibroblasts [28], $\mathrm{CD} 90^{+}$activated endothelium [29] and sublining $\mathrm{CD} 163^{+}$macrophages.

\section{Activin A, but not GM-CSF, is increased in synovial fluid from all arthritis groups.}

Analysis of paired synovial fluid (SF) samples from patients with persistent UA $(n=12), U A>R A(n=9), U A>$ PsA $(n=7), \operatorname{RA}(n=9)$ and PsA $(n=9)$ indicated that GM-CSF levels were below detection levels in most SF samples, except for 4 out of $9(44 \%)$ persistent UA patients who were csDMARD naïve, and one UA > PsA patient out of 7 analyzed (14\%), who had increased GM-CSF levels (Fig. 1B). We also measured the level of activin $A$, which plays an important role in the early phases of the inflammatory process [30]. By contrast to GM-CSF, high levels of activin A were detected in all SF samples, with concentrations ranging from 0.2 to $72 \mathrm{ng} / \mathrm{ml}$, with UA > RA and RA samples expressing higher levels than all other groups (Fig. 1C). Taken together, our results suggest that ST GM-CSF and SF activin A are detected in both the undifferentiated and established phases of arthritis, but with significantly higher levels of activin $A$ in the undifferentiated and established phases of RA. 
Synovial tissue CD163 + macrophages from patients with undifferentiated and established arthritis exhibit a predominantly GM-CSF-dependent pro-inflammatory polarization state

GSEA to compare RA macrophages and monocyte-derived macrophage transcriptome [25] revealed positive enrichment of pro-inflammatory GM-CSF-conditioned monocyte-derived macrophages (GM-M $\emptyset$ )-specific genes in RA macrophages (Fig. 2A). In fact, the leading edge of the GSEA revealed the genes encoding activin A (INHBA), the metalloproteinase MMP12 and the chemokine CCL17 [31] (Fig. 2A).

Since our results indicated that ST in UA show enriched expression of GM-CSF in the stromal cells and activin $A$ in the SF, we next determined the expression of proteins associated with GM-CSF-driven polarization (activin A, MMP12 and TNFa) and M-CSF-driven polarization (CD209) [17] (Fig. 2B) in ST CD163+ macrophages of persistent UA, UA > RA, UA > PsA, and in established RA and PsA patients (Fig. 3A). Similar to active established RA [17], activin A, TNFa and MMP12 were readily found in $C D 163^{+}$macrophages within the synovial sublining of UA, either evolving or not (Fig. $3 A$ ), and essentially the same results were obtained in PsA, where ST CD163 + macrophages expressed GM-CSF-dependent polarization markers (Fig. 3A). By contrast, CD163 + macrophages from healthy synovium did not express TNFa, MMP12 or activin A (Fig. 3A). Quantification of the above indicated markers revealed that $C D 163^{+}$macrophages from UA > RA and UA > PsA exhibited similar expression levels of activin A, TNFa and MMP12 as CD163 ${ }^{+}$macrophages from established RA and PsA, and higher levels than CD163 + macrophages from healthy synovium (Supplementary Fig. 1, Fig. 3B). MMP12 expression was significantly higher in persistent UA than in UA > RA, although no significant differences in markers of systemic inflammation or in the percentage of patients receiving csDMARDs were found between the two UA groups (Table 1). Therefore, the expression of activin A, MMP12 and TNFa designates the GM-CSF-dependent pro-inflammatory state of CD $163^{+}$ST macrophages in undifferentiated and established arthritis (Fig. 3A-B).

Two subsets of $\mathrm{CD} 163{ }^{+} \mathrm{CD} 209$ macrophages are found in the synovial tissue from patients with undifferentiated and established arthritis

With respect to the M-CSF-associated marker CD209, two subsets of $C D 163^{+}$macrophages were found in the synovial sublining of UA and established arthritis patients (CD163 ${ }^{+} \mathrm{CD} 209^{+}$and $\left.\mathrm{CD} 163^{+} \mathrm{CD} 209^{\text {low/ }}{ }^{-}\right)$, which differed in their respective MFI for CD209 fluorescence (MFI > 34 for the $\mathrm{CD} 163^{+} \mathrm{CD} 209^{+}$subset, and MFI $<34$ for the $\mathrm{CD} 163^{+} \mathrm{CD} 209^{\text {low/- }}$ subset) (Fig. 4A-B). Synovial tissue from healthy controls also showed these two macrophage subsets, with a higher percentage of $\mathrm{CD} 163^{+} \mathrm{CD} 209^{+}$cells (80\%) than that found in UA (Fig. 4C). Furthermore, the percentage of the $\mathrm{CD} 163^{+} \mathrm{CD} 209^{+}$macrophage subset in PSA ST was significantly higher than in that from persistent UA (Fig. 4B-C, Supplementary Fig. 2). Taken together, these findings suggest that $\mathrm{CD} 163^{+} \mathrm{CD} 209^{+}$are resident macrophages with a potential anti-inflammatory function. However, both macrophage subsets (CD209+ and $\mathrm{CD} 209^{\text {low/- }}{ }^{-}$) expressed the proinflammatory markers activin A, TNFa and MMP12 in all patients from the UA and established disease groups (Supplementary Fig. 2).

\section{Macrophage density discriminates undifferentiated from established arthritis}


We determined macrophage density in ST from patients with persistent UA, UA > RA, UA > PsA, established RA and PsA, and healthy controls. CD163 and CD115 were used to gate ST macrophages as they are expressed by most macrophages (Supplementary Fig. 3) [32]. Macrophage density was significantly higher in synovium from patients with inflammatory joint disease compared with healthy controls (Fig. 5A-B) and was comparable between both UA evolving groups, with 650 macrophages/mm² in UA > RA and 649 macrophages $/ \mathrm{mm}^{2}$ in UA > PsA, but significantly higher than in established RA and PsA, respectively (Fig. 5A). Synovial tissue from persistent UA showed significantly lower macrophage density than UA $>$ RA and UA > PsA (Fig. 5A). These results suggest that the initial phases of RA and PsA are characterized by a significantly higher density of $\mathrm{CD} 163^{+}$macrophages compared with persistent UA and established disease. Since all groups had a similar proportion of patients on csDMARD therapy, differences in macrophage density could not be attributed to differences in treatment. In support of this rationale, more UA > PsA patients had received csDMARDs compared with PsA patients. However, 56\% of patients with persistent UA were csDMARD-naïve, and had significantly lower $\mathrm{CD}_{163^{+}}$macrophage density than UA > RA, UA > PsA, established RA or PsA (Table 1).

\section{Discussion}

ST macrophages are key producers of cytokines relevant to the physiopathology of chronic arthritis and changes in them correlate with disease activity, radiographic progression and the response to therapy [1013]. However, macrophages are composed of a heterogeneous population with different functional profiles: pro-inflammatory, anti-inflammatory or reparative [33]. Macrophage polarization has been demonstrated in experimental models of immune-mediated inflammatory diseases and in human cancer [34].

The characterization of ex-vivo CD14 ${ }^{+}$macrophages isolated from synovial fluid of patients with active RA indicate that they exhibit a transcriptomic and protein profile compatible with GM-CSF-skewed macrophage polarization [17]. Activin A and other proteins encoded by several GM-CSF-associated gene markers, including MMP12 and CCR2, have been also detected in macrophages from active RA joints [17-19]. GM-CSF induces macrophage differentiation and survival from hematopoietic progenitor cells [31, 35]. In contrast to M-CSF, GM-CSF is undetectable in the systemic circulation during homeostasis but is produced and active at sites of tissue inflammation, and is a key driver of tissue inflammation and arthritic pain [36, 37]. GM-CSF was also pathogenic in experimental models of SpA [38] and is emerging as a central player in chronic inflammatory diseases, including mouse models of multiple sclerosis [39], bowel inflammation [40], and RA [41]. GM-CSF signals through GM-CSFR, priming myeloid cells to produce inflammatory mediators that promote the activation of synovial fibroblasts, activation of the vasculature and differentiation of effector $\mathrm{T}$ cells. Activated synovial fibroblasts secrete GM-CSF in response to inflammatory mediators, thus implying that GMCSF is a communication conduit between the innate and adaptive immunity critically-involved in chronic inflammation [36].

The GM-CSF receptor was reported to be overexpressed in CD68 + and CD163 ${ }^{+}$macrophages from the ST of RA and PsA patients compared with osteoarthritis patients and healthy controls [42]. Clinical trials targeting GM-CSF or GM-CSF receptor-a in RA have shown sustained clinical responses without major safety concerns $[43,44]$. 
To our knowledge, this is the first study assessing GM-CSF expression and cellular source, macrophage polarization state and density in the ST of the most prevalent immune-mediated inflammatory joint diseases, RA and PsA, and their undifferentiated phases. We found that the main cellular sources of GM-CSF in the synovium of all chronic arthritis groups studied are stromal cells and $\mathrm{CD} 163^{+}$macrophages. Sublining $\mathrm{CD} 90^{+}$ $\mathrm{FAP}^{+}$synovial fibroblasts, the only subtype reported as pathogenic, which is specifically increased in RA compared with osteoarthritis [28] and $\mathrm{CD} 90^{+}$activated endothelium [29] show high GM-CSF expression. Although intracellular GM-CSF expression by ST polyfunctional lymphocytes (Th1, Th17 and ex-Th17 subtypes) have been reported using flow cytometry in PsA and the number of these cells correlated with the PsA disease activity score (DAPSA) [45], we could not confirm GM-CSF expression by $\mathrm{CD}^{+}$or $\mathrm{CD}^{+}{ }^{+} \mathrm{T}$ cells in our samples. No expression of GM-CSF on healthy synovium was found.

Among the GM-CSF-derived macrophage markers, we detected activin A both in the synovial stroma and synovial fluid of all UA and established arthritis patients, although higher levels of SF activin A were found in RA and its undifferentiated phase. Activin $\mathrm{A}$ is a member of the TGF $\beta$ factor family encoded by the INHBA gene and mediates, in part, the macrophage-polarizing ability of RA synovial fluid [17, 46]. Activin A contributes to the proinflammatory macrophage polarization triggered by GM-CSF and limits acquisition of the anti-inflammatory phenotype [18]. Activin $A$ is elevated in the early stages of other inflammatory diseases and exerts pro- or anti-inflammatory activities depending on its concentration and cellular context [47]. In the particular case of the undifferentiated and established arthritis in our study, activin A levels fell within the proinflammatory side [47], stressing its contribution to the polarization of macrophages within the inflamed synovium.

Despite the high and generalized expression of GM-CSF in the synovium of all arthritis groups analyzed, only a few csDMARD naïve patients with non-evolving UA had detectable levels in synovial fluid. This finding was expected, as it is known that GM-CSF is mainly expressed in inflamed tissue.

Our results confirm our previous results in RA [17] and extend them to PsA synovitis and their respective UA phases, including persistent UA, suggesting that the pattern of polarization of ST macrophages, mainly proinflammatory, is similar and is already present in all phases of these chronic inflammatory joint diseases. A previous study analyzing macrophage subsets in human arthritis found M1 polarization predominating in RA and IL-10 expressing macrophages in SpA, including PsA [48].

Despite the pathogenic differences between RA and PsA, we found similar patterns of expression of proteins induced by GM-CSF and M-CSF in $\mathrm{CD}_{163^{+}}$macrophages of PsA, RA and their undifferentiated phases. Although PSA ST had significantly more $\mathrm{CD} 163^{+}$expressing the anti-inflammatory CD209 marker, which

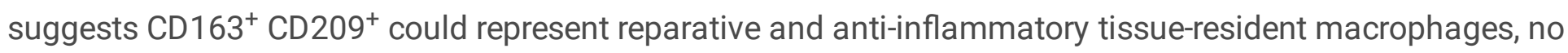
differences were found in the expression of proinflammatory markers (activin A, TNF, MMP-12) between CD $163^{+}$macrophages CD209 ${ }^{+}$and CD209low/- .

Our results show that the density of CD163 + macrophages is significantly higher in UA evolving to RA or PsA compared with established disease or persistent UA. This is an unexpected finding as more UA > PsA patients had received csDMARDs than PsA patients. However, $56 \%$ of persistent UA patients were csDMARD-naïve

Page $11 / 23$ 
and had significantly lower CD163 + macrophage density than UA > RA, UA > PsA, or established RA or PsA patients. Although cautiously, these findings suggest that a significant increase in ST CD163 + macrophage density could be a marker of transition from undifferentiated to definite disease, both in RA and PsA. In fact, a study searching for ST predictors of clinical differentiation in patients with seronegative undifferentiated peripheral arthritis reported that 6 out of 42 patients with a definite diagnosis after one year of follow up (2 RA, 2 SpA, 2 PsA) had higher histological CD 68 + macrophage scores than patients persisting with undifferentiated arthritis [49]

Our study has some limitations, including the retrospective design and the relatively small sample size. Although some patients were receiving csDMARDs (mainly methotrexate), which could modify our findings, this factor was comparable in all groups. The study has the strength of the follow-up of UA patients until they met RA or PsA classification criteria, the group of persistent UA patients (treated or not with csDMARDs) as positive controls and the ST from healthy controls. Likewise, the results were consistent for all types of arthritis.

\section{Conclusion}

Our results show a high, generalized expression of GM-CSF in stromal cells and sublining CD $163^{+}$ macrophages from synovial tissue of UA patients evolving or not to RA or PsA compared with healthy controls. $\mathrm{CD}_{163^{+}}$macrophages expressed a pro-inflammatory profile of biomarkers (activin A, TNFa and MMP-12) in synovial tissue from all arthritis subtypes studied, while in paired samples of synovial fluid only activin A levels were widely detected. $\mathrm{CD} 163^{+}$macrophages were significantly higher in the UA phases of RA and PsA compared with established disease. Taken together, our findings support the concept that GM-CSF is a strong driver of macrophage polarization and a potential therapeutic target, not only in RA but also in PsA and in UA. We also found that the ST sublining of undifferentiated and established arthritis patients showed a high percentage of $\mathrm{CD} 163^{+} \mathrm{CD} 209^{\text {low/- }}$ whereas healthy controls exhibited a high percentage of $\mathrm{CD} 163^{+}$ $\mathrm{CD}_{209^{+}}$, suggesting they may be resident macrophages with a potential anti-inflammatory function.

\section{Abbreviations}

ACPA Anti-citrullinated protein antibodies

csDMARD Conventional synthetic disease-modifying antirheumatic drug

CRP C-Reactive Protein

DAPI 4',6-diamidino-2-phenylindole

DMARDs Disease-modifying antirheumatic drugs

ESR Erythrocyte sedimentation rate

FAP Fibroblast activation protein

Page $12 / 23$ 
GM-CSF Granulocyte-macrophage colony-stimulating factor

GM-CSFR Granulocyte-macrophage colony stimulating factor receptor low-affinity subunit

INHBA Inhibin subunit beta A

MFI Mean fluorescence intensity

MMP12 Matrix metalloproteinase 12

PsA Psoriatic arthritis

RA Rheumatoid arthritis

RF Rheumatoid factor

SEM Standard Error of Mean

SF Synovial fluid

SJC Swollen joint count

SL Sublining

SpA Spondyloarthropathy

ST Synovial tissue

TJC Tender joint count

TNFa Tumor necrosis factor alpha

UA Undifferentiated arthritis

\section{Declarations}

\section{Ethics approval and consent to participate:}

This study was conducted in accordance with the Declaration of Helsinki and was approved by the Hospital Clínic of Barcelona Clinical Research Ethics Committee (2014/0398). Signed informed consent was obtained from all patients.

Consent for publication:

Not applicable

Availability of data and material: 
The datasets generated and/or analyzed for the present study are available from the corresponding author on reasonable request.

\section{Competing interests:}

The authors declare they have no competing interests.

\section{Funding:}

This work was supported by grants PI17/00037 from Instituto de Salud Carlos III/FEDER to APK, grants PI14/00785 and PI17/00993 from Instituto de Salud Carlos III to JDC, grant RIER RD16/0012/0010 and RD16/0012/0007 FEDER to JDC and APK, respectively. FEDER, Fondo Europeo de Desarrollo Regional: una manera de hacer Europa. SFR and APK are supported by FIBHGM. AC received grants from the Hospital Clinic of Barcelona "Emili Letang 2014", and the Catalan Society of Rheumatology.

\section{Authors' contributions:}

APK and JDC had full access to all the data in the study and take responsibility for the integrity of the data and the accuracy of data analysis. APK and JDC were responsible for the study design. AC, RC and JR performed clinical data acquisition. SFR, LEC, RS, RGC and APK were responsible for the analysis. SFR, LEC, AC, APK and JDC performed the data interpretation. Manuscript preparation was by SFR, LEC, AC, APK and JDC. All authors read and approved the final manuscript.

\section{Acknowledgements}

The authors acknowledge Julia Villarejo for technical help, Angel L. Corbí for helpful discussions, and David Buss for technical assistance.

\section{References}

1. van der Horst-Bruinsma IE, Speyer I, Visser H, Breedveld FC, Hazes JM. Diagnosis and course of earlyonset arthritis: results of a special early arthritis clinic compared to routine patient care. $\mathrm{Br} \mathrm{J}$ Rheumatol. 1998;37:1084-8.

2. Wolfe F, Ross K, Hawley DJ, Roberts FK, Cathey MA. The prognosis of rheumatoid arthritis and undifferentiated polyarthritis syndrome in the clinic: a study of 1141 patients. $J$ Rheumatoloy. 1993;20:2005-9.

3. Olivieri I, Sarzi-Puttini P, Bugatti S, Atzeni F, d'Angelo S, Caporali R. Early treatment in early undifferentiated arthritis. Autoimmun Rev. 2012;11:589-92.

4. Quinn MA, Green MJ, Marzo-Ortega H, Proudman S, Karim Z, Wakefield RJ, et al. Prognostic factors in a large cohort of patients with early undifferentiated inflammatory arthritis after application of a structured management protocol. Arthritis Rheum. 2003;48:3039-45. 
5. Paramarta JE, Baeten D. Spondyloarthritis. From Unifying Concepts to Improved Treatment. Rheumatology. 2014;53:1547-59.

6. Celis R, Cuervo A, Ramírez J, Cañete JD. Psoriatic arthritis: singularity and clinical implications. Front Med (Lausanne). 2019 Feb;11:6:14.

7. Veale DJ, Fearon U. What makes psoriatic and rheumatoid arthritis so different? RMD Open. 2015; 28;1(1):e000025.

8. Orr C, Vieira-Sousa E, Boyle DL, Buch MH, Buckley CD, Cañete JD, et al. Synovial tissue research: a stateof-the-art review. Nat Rev Rheumatol. 2017;13:463-75.

9. Cañete JD, Santiago B, Cantaert T, Sanmartí R, Palacin A, Celis R, et al. Ectopic lymphoid neogenesis in psoriatic arthritis. Ann Rheum Dis. 2007;66:720-6.

10. Mulherin D, Fitzgerald O, Bresnihan B. Synovial tissue macrophage populations and articular damage in rheumatoid arthritis. Arthritis Rheum. 1996;39:115-24.

11. Ritchlin CT, Haas-Smith SA, Li P, Hicks DG, Schwarz EM. Mechanisms of TNF-alpha- and RANKLmediated osteoclastogenesis and bone resorption in psoriatic arthritis. J Clin Invest. 2003;111:821-31.

12. Haringman JJ, Gerlag DM, Zwinderman AH, Smeets TJ, Kraan MC, Baeten D, et al. Synovial tissue macrophages: a sensitive biomarker for response to treatment in patients with rheumatoid arthritis. Ann Rheum Dis. 2005;64:834-8.

13. Baeten D, Kruithof E, De Rycke L, Boots AM, Mielants H, Veys EM, et al. Infiltration of the synovial membrane with macrophage subsets and polymorphonuclear cells reflects global disease activity in spondyloarthropathy. Arthritis Res Ther. 2005;7:R359-69.

14. Kruithof E, Baeten D, De Rycke L, Vandooren B, Foell D, Roth J, et al. Synovial histopathology of psoriatic arthritis, both oligo- and polyarticular, resembles spondyloarthropathy more than it does rheumatoid arthritis. Arthritis Res Ther. 2005;7:R569-80.

15. Salvador G, Sanmarti R, García-Peiró A, Rodríguez-Cros JR, Muñoz-Gómez J, Cañete JD. p53 Expression in Rheumatoid and Psoriatic Arthritis Synovial Tissue and Association With Joint Damage. Ann Rheum Dis. 2005;64:183-7.

16. Vandooren B, Noordenbos T, Ambarus C, Krausz S, Cantaert T, Yeremenko N, et al. Absence of a classically activated macrophage cytokine signature in peripheral spondylarthritis, including psoriatic arthritis. Arthritis Rheum. 2009;60:966-75.

17. Soler Palacios B, Estrada-Capetillo L, Izquierdo E, Criado G, Nieto C, Municio C, et al. Macrophages from the synovium of active rheumatoid arthritis exhibit an activin A-dependent pro-inflammatory profile. $J$ Pathol. 2015;235:515-26.

18. Sierra-Filardi E, Puig-Kröger A, Blanco FJ, Nieto C, Bragado R, Palomero MI, et al. Activin A skews macrophage polarization by promoting a proinflammatory phenotype and inhibiting the acquisition of anti-inflammatory macrophage markers. Blood. 2011;117(19):5092-101.

19. Puig-Kröger A, Sierra-Filardi E, Domínguez-Soto A, Samaniego R, Corcuera MT, Gómez-Aguado F, et al. Folate receptor beta is expressed by tumor-associated macrophages and constitutes a marker for $\mathrm{M} 2$ anti-inflammatory/regulatory macrophages. Cancer Res. 2009;69(24):9395-403. 
20. Campbell IK, Rich MJ, Bischof RJ, Hamilton JA. The colony-stimulating factors and collagen-induced arthritis: exacerbation of disease by M-CSF and G-CSF and requirement for endogenous M-CSF. J Leukoc Biol. 2000;68:144-50.

21. Al-Mossawi MH, Chen L, Fang H, Ridley A, de Wit J, Yager N, et al. Unique transcriptome signatures and GM-CSF expression in lymphocytes from patients with spondyloarthritis. Nat Commun. 2017;8:1510.

22. Cook AD, Braine EL, Campbell IK, Rich MJ, Hamilton JA. Blockade of collagen induced arthritis postonset by antibody to granulocyte-macrophage colony-stimulating factor (GM-CSF): requirement for GMCSF in the effector phase of disease. Arthritis Res. 2001;3:293-8.

23. Arnett FC, Edworthy SM, Bloch DA, McShane DJ, Fries JF, Cooper NS, et al. The American Rheumatism Association 1987 revised criteria for the classification of rheumatoid arthritis. Arthritis Rheum. 1988;31:315-24.

24. Taylor W, Gladman D, Helliwell P, Marchesoni A, Mease P, Mielants H, CASPAR Study Group. Classification criteria for psoriatic arthritis - Development of new criteria from a large international study. Arthritis Rheum. 2006;54:2665-73.

25. Subramanian A, Tamayo P, Mootha VK, Mukherjee S, Ebert BL, Gillette MA, et al. Gene set enrichment analysis: a knowledge-based approach for interpreting genome-wide expression profiles. Proc Natl Acad Sci U S A. 2005;102(43):15545-50.

26. Yarilina A, Park-Min KH, Antoniv T, Hu X, Ibashkiv LB. TNF activates an IRF1-dependent autocrine loop leading to sustained expression of chemokines and STAT1-dependent type I interferon-response genes. Nat Immunol. 2008;9(4):378-87.

27. Gonzalez-Dominguez E, Dominguez-Soto A, Nieto C, Flores-Sevilla JL, Pacheco-Blanco M, Campos-Pena $\mathrm{V}$, et al. Atypical activin A and IL-10 production impairs human CD16 + monocyte differentiation into antiinflammatory macrophages. J Immunol. 2016;196:1327-37.

28. Mizoguchi F, Slowikowski K, Wei K, Marshall JL, Rao DA, Chang SK, et al. Functionally distinct diseaseassociated fibroblast subsets in rheumatoid arthritis. Nat Commun. 2018;9:789.

29. Wandel E, Saalbach A, Sittig D, Gebhardt C, Aust G. Thy-1 (CD90) is an interacting partner for CD97 on activated endothelial cells. J Immunol. 2012;188:1442-50.

30. Domínguez-Soto A, Sierra-Filardi E, Puig-Kröger A, Pérez-Maceda B, Gómez-Aguado F, Corcuera MT, Sánchez-Mateos P, Corbí AL. Dendritic cell-specific ICAM-3-grabbing nonintegrin expression on M2polarized and tumor-associated macrophages is macrophage-CSF dependent and enhanced by tumorderived IL-6 and IL-10. J Immunol. 2011;186(4):2192-200.

31. Hamilton JA. GM-CSF in inflammation. J Exp Med. 2020;217(1):e20190945. doi:10.1084/jem.20190945.

32. Mosser DM, Edwards JP. Exploring the full spectrum of macrophage activation. Nat Rev Immunol. 2008;8:958-69.

33. Culemann S, Grüneboom A, Nicolás-Ávila J, Weidner D, Lämmle KF, Rothe T, et al. Locally Renewing Resident Synovial Macrophages Provide a Protective Barrier for the Joint. Nature. 2019;572(7771):6705.

34. Wynn TA, Chawla A, Pollard JW. Macrophage biology in development, homeostasis and disease. Nature. 2013;496:445-55. 
35. Burgess AW, Metcalf $D$. The nature and action of granulocyte-macrophage colony stimulating factors. Blood. 1980;56:947-58.

36. Becher B, Tugues S, Greter M. GM-CSF: From Growth Factor to Central Mediator of Tissue Inflammation. Immunity. 2016;45:963-73.

37. Cook AD, Pobjoy J, Sarros S, Steidl S, Dürr M, Lacey DC, et al. Granulocyte-macrophage colonystimulating factor is a key mediator in inflammatory and arthritic pain. Ann Rheum Dis. 2013;72:265-70.

38. Regan-Komito D, Swann JW, Demetriou P, Cohen ES, Horwood NJ, Sansom SN, et al. GM-CSF Drives Dysregulated Hematopoietic Stem Cell Activity and Pathogenic Extramedullary Myelopoiesis in Experimental Spondyloarthritis. Nat Commun. 2020;11(1):155.

39. Monaghan KL, Wan ECK. The Role of Granulocyte-Macrophage Colony-Stimulating Factor in Murine Models of Multiple Sclerosis. Cells. 2020;9(3):611.

40. Egea L, Hirata Y, Kagnoff MF. GM-CSF: a role in immune and inflammatory reactions in the intestine. Expert Rev Gastroenterol Hepatol. 2010;4:723-31.

41. Hirota K, Hashimoto M, Ito Y, Matsuura M, Ito H, Tanaka M, et al. Autoimmune Th17 Cells Induced Synovial Stromal and Innate Lymphoid Cell Secretion of the Cytokine GM-CSF to Initiate and Augment Autoimmune Arthritis. Immunity. 2018;48:1220-32.

42. Greven DE, Cohen ES, Gerlag DM, Campbell J, Woods J, Davis N, et al. Preclinical Characterisation of the GM-CSF Receptor as a Therapeutic Target in Rheumatoid Arthritis. Ann Rheum Dis. 2015;74:1924-30.

43. Cook AD, Hamilton JA. Investigational therapies targeting the granulocyte macrophage colonystimulating factor receptor-a in rheumatoid arthritis: focus on mavrilimumab. Ther Adv Musculoskelet Dis. 2018;10:29-383.

44. Burmester GR, McInnes IB, Kremer JM, Miranda P, Vencovský J, Godwood A, et al. Mavrilimumab, a Fully Human Granulocyte-Macrophage Colony-Stimulating Factor Receptor a Monoclonal Antibody: LongTerm Safety and Efficacy in Patients With Rheumatoid Arthritis. Arthritis Rheumatol. 2018;70:679-89.

45. Wade SM, Canavan M, McGarry T, Low C, Wade SC, Mullan RH, et al. Association of Synovial Tissue Polyfunctional T-cells With DAPSA in Psoriatic Arthritis. Ann Rheum Dis. 2019;78:350-4.

46. Massague J. TGF $\beta$ signalling in context. Nat Rev Mol Cell Biol. 2012;13:616-30.

47. Dong F, He X. Activin A. A Potential Therapeutic Target for Characterizing and Stopping Joint Pain Early in Rheumatoid Arthritis Patients. Inflammation. 2014;37:170-6.

48. Ambarus CA, Noordenbos T, de Hair MJ, Tak PP, Baeten DL. Intimal lining layer macrophages but not synovial sublining macrophages display anIL-10 polarized-like phenotype in chronic synovitis. Arthritis Res Ther. 2012;14:R74.

49. Alivernini S, Tolusso B, Petricca L, Bui L, Di Mario C, Gigante MR, et al et al. Synovial Predictors of Differentiation to Definite Arthritis in Patients With Seronegative Undifferentiated Peripheral Inflammatory Arthritis: microRNA Signature, Histological, and Ultrasound Features. Front Med (Lausanne). 2018;5:186.

\section{Figures}




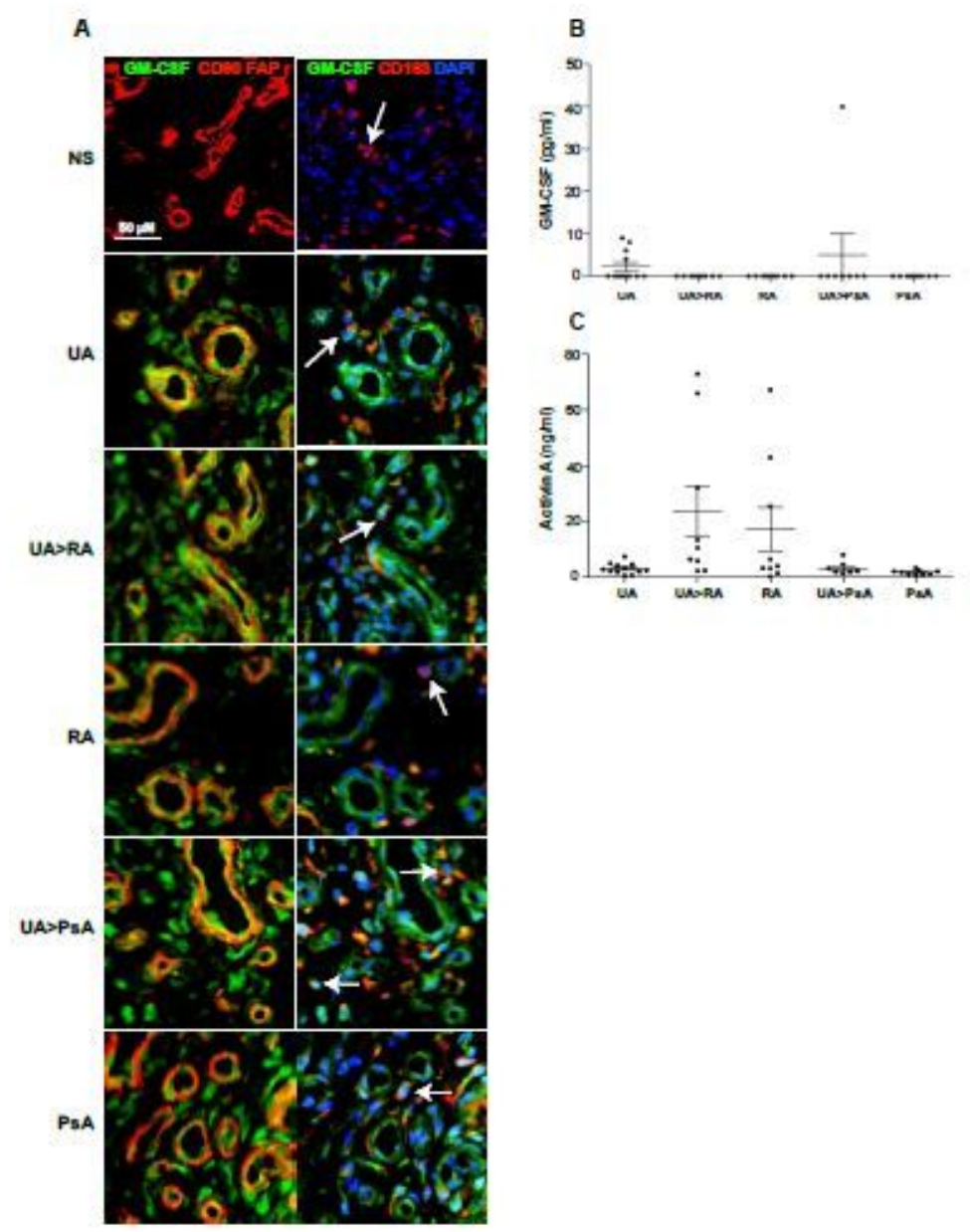

Figure 1

GM-CSF expression in stromal cells from synovial tissue of undifferentiated arthritis patients. A. Immunofluorescent analysis of normal synovium (NS), persistent undifferentiated arthritis (UA), undifferentiated arthritis evolving to RA (UA>RA), rheumatoid arthritis (RA), undifferentiated arthritis evolving to psoriatic arthritis (UA>PsA) and psoriatic arthritis (PsA) synovial tissues (ST) determined by confocal microscopy using anti-GM-CSF (green), anti-CD90, anti-FAP or anti-CD163 (red)-specific antibodies; nuclei were counterstained with DAPI. Samples were devoid of staining when incubated with isotype-matched irrelevant antibodies as negative controls. The experiment was carried out in independent samples from each type (NS, $n=6 ; U A, n=16 ; U A>R A, n=8 ; R A, n=12 ; U A>P s A, n=9$; and PsA, $n=10$ ) and representative experiments are shown. $C D 163+$ macrophages are indicated by white arrows.Scale bars, $50 \mu \mathrm{m}$. Determination of levels of GM-CSF (B)and activin A(C) in the synovial fluid of patients with UA $(n=12), U A>R A(n=9), R A(n=9), U A-P s A$ $(n=7)$, and PsA $(n=9)$, as determined by ELISA. 
A

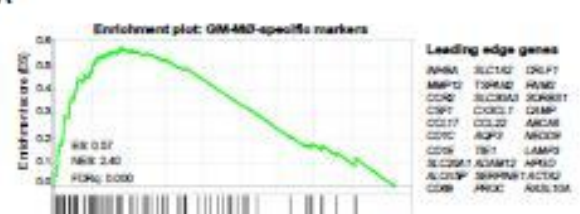

|||||||||||||||||| $\mid$

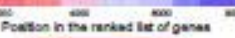

(h)

B
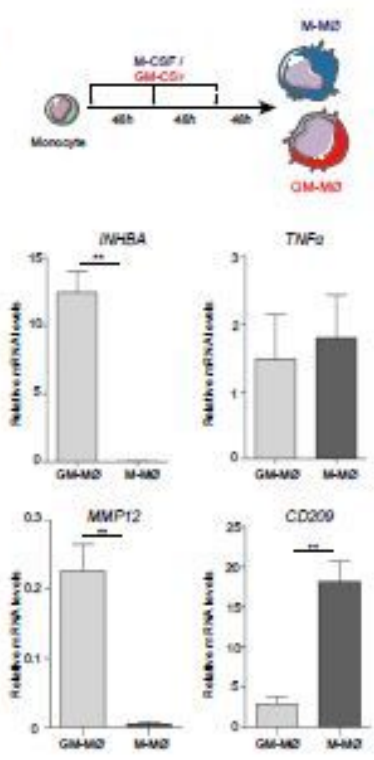

Figure 2

Expression of polarization markers by RA macrophages and in-vitro generated monocyte-derived macrophages.A. Gene set enrichment analysis (GSEA) on the ranked list of genes obtained from the comparison of the transcriptome of RA macrophages (RA mac) versus monocyte-derived macrophages (GSE10500) using the set of genes with the highest GM-CSF-induced upregulation in monocyte-derived macrophages differentiated with GM-CSF. B. INHBA, MMP12, CD209 and TNFA mRNA expression levels determined by qRT-PCR on monocytes differentiated with GM-CSF (GM-M $\varnothing$ ) and M-CSF (M-M $\emptyset$ ). Mean \pm SEM of four independent donors are shown $\left({ }^{*} p<0.05,{ }^{*} \mathrm{p}<0.01\right.$, Student $t$ test $)$. 


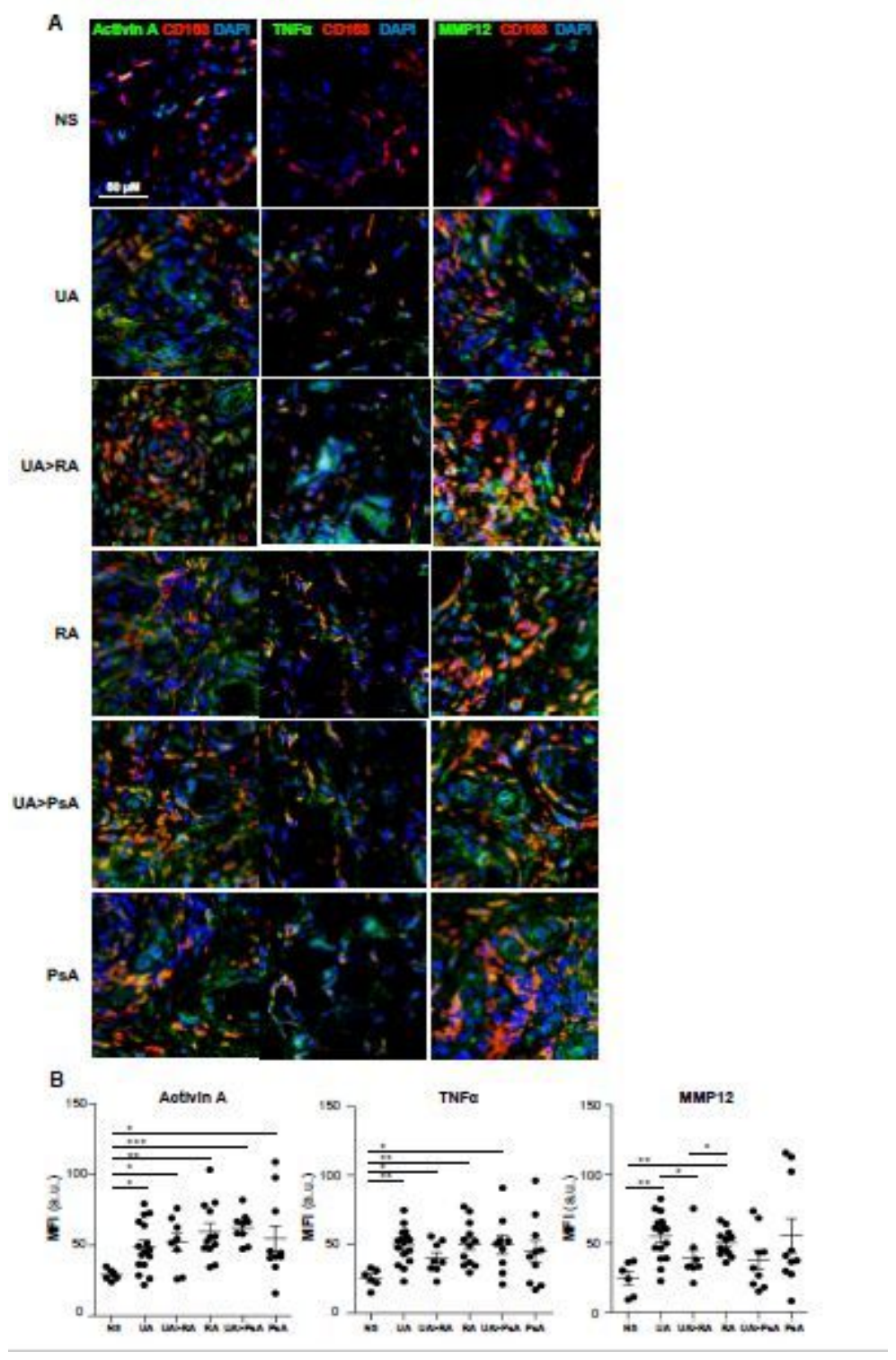

\section{Figure 3}

Expression of macrophage-pro-inflammatory polarization markers by CD163+ macrophages from undifferentiated arthritis patients.A. Immunofluorescent analysis of synovial tissues as determined by confocal microscopy using anti-activin A, TNFa-, MMP12-specific antibodies; nuclei were counterstained with DAPI. Samples were devoid of staining when incubated with isotype-matched irrelevant antibodies as negative controls. The experiment was carried out in independent samples from each type (NS, $n=6 ; U A, n=16$; $U A>R A, n=8 ; R A, n=12 ; U A>P s A, n=9$; and PsA, $n=10$ ) and representative experiments are shown. Scale bars, $50 \mu \mathrm{m}$ B. Summary dot plot showing mean MFI values of activin A, TNF-a and MMP12 expression in CD163+ macrophages from NS, UA, UA>RA, RA, UA>PsA, and PsA synovial tissues samples. Mean \pm SEM are shown ( ${ }^{*} p<0.05,{ }^{*} \mathrm{p}<0.01$, Mann-Whitney test). 
A
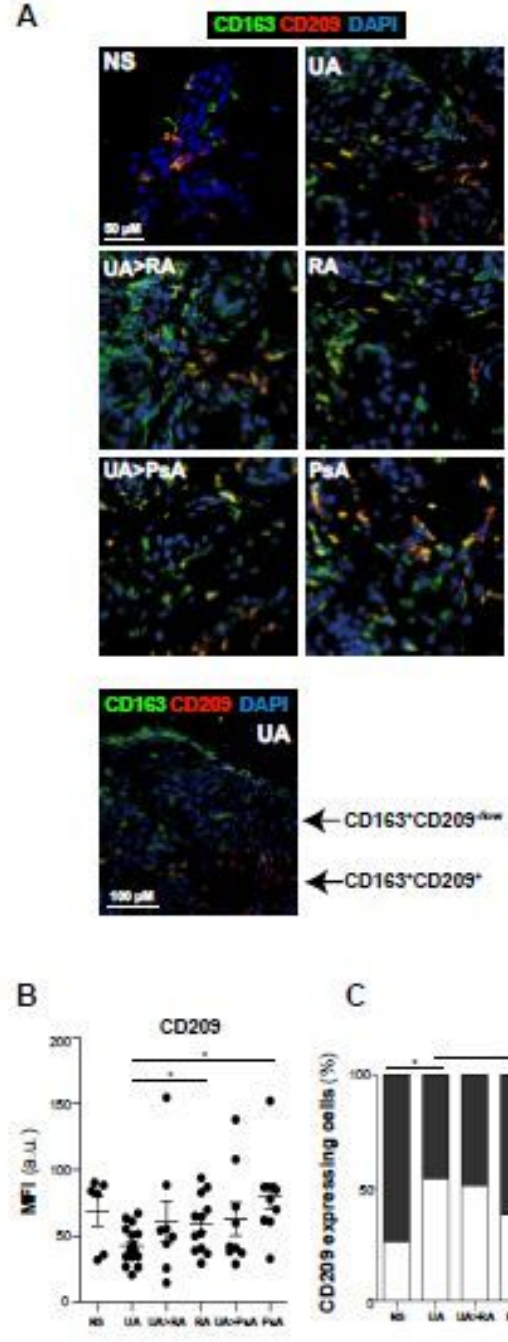

C

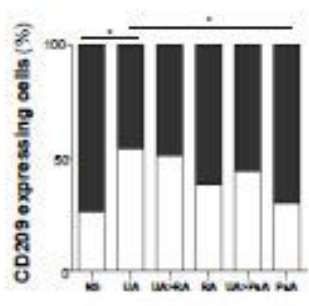

\section{Figure 4}

CD163+ CD209 macrophage subsets in synovial tissueA.CD163 (green) and CD209 (red) staining of NS, UA, UA-RA, RA, UA-PsA and PsA synovial tissues. Scale bar, as shown. B. Summary dot plot showing mean MFI values of CD209 expression in CD163+ macrophages from synovial tissue samples. The experiment was carried out in independent samples from each type (NS, $n=6$; UA, $n=16$; UA>RA, $n=8 ; R A, n=12 ; U A>P s A, n=9$; and PsA, $n=10)$ and representative experiments are shown. Mean $\pm S E M$ are shown $\left({ }^{*} p<0.05,{ }^{*} p<0.01\right.$, MannWhitney test). C. Percentage of CD209-expressing macrophages in NS, UA, UA>RA, RA, UA>PsA and PsA synovial tissues. The MFI values of 8-12 tissues samples of each group were used for classification as "low/neg” (white) or "high" (> 34 a.u., black) CD209 expression. 
A

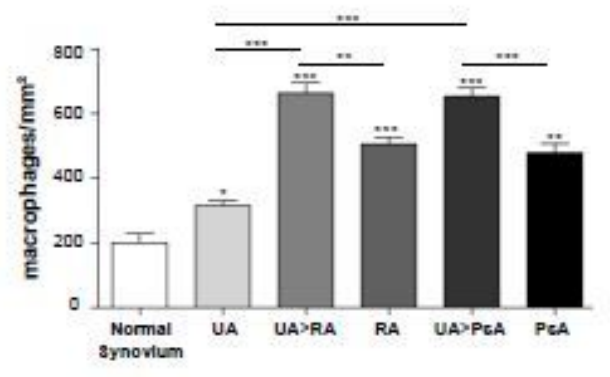

B

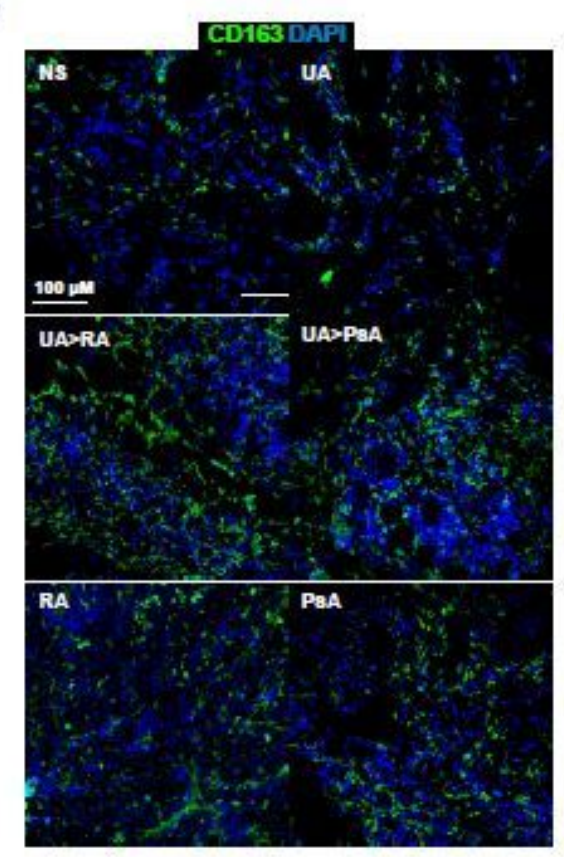

\section{Figure 5}

Macrophage density in normal, undifferentiated arthritis, rheumatoid arthritis and psoriatic arthritis synovial tissue. A. Quantification of CD163+ macrophages from normal $(N S, n=6)$, undifferentiated arthritis (UA, $n=16)$, undifferentiated arthritis evolving to $R A(U A>R A, n=8)$, undifferentiated arthritis becoming psoriatic arthritis (UA>PsA, $n=9)$, rheumatoid arthritis ( $R A, n=12)$ and psoriatic arthritis (PsA, $n=10)$ synovial tissues. The total number of macrophages was normalized based on selected tissue area (mm2). Mean \pm SEM is shown. Significant differences are indicated ( ${ }^{*} p<0.05,{ }^{* \star} p<0.01,{ }^{* \star \star} p<0.001$, Mann-Whitney test). B. Immunofluorescent analysis of NS, UA, UA>RA, RA, UA>PsA, and PsA synovial tissues as determined by confocal microscopy using anti-CD163 specific antibody (green); nuclei were counterstained with DAPI (blue). The experiment was carried out in independent samples from each type (6 normal synovial tissue controls and 8-16 remaining synovial tissues) and representative experiments are shown.Scale bars, $100 \mu \mathrm{m}$.

\section{Supplementary Files}

This is a list of supplementary files associated with this preprint. Click to download. 
- SupplementaryFigure325520.pdf

- SupplementaryFigure125520.pdf

- SupplementaryFigure225520.pdf

- SUPPLEMENTARYFIGURES.docx 